\title{
New directions in point-contact spectroscopy based on scanning tunneling microscopy techniques (Review Article)
}

\author{
E. Tartaglini ${ }^{1}$, T.G.A. Verhagen ${ }^{1}$, F. Galli ${ }^{1}$, M.L. Trouwborst ${ }^{1}$, \\ R. Müller ${ }^{2}$, T. Shiota $^{3}$, J. Aarts ${ }^{1}$, and J.M. van Ruitenbeek ${ }^{1}$ \\ ${ }^{1}$ Kamerlingh Onnes Laboratorium, Universiteit Leiden, PO Box 9504, 2300 RA Leiden, The Netherlands \\ E-mail: aarts@physics.leidenuniv.nl \\ ruitenbeek@physics.leidenuniv.nl \\ ${ }^{2}$ University of Konstanz, FB Physik, 10 Universitätstrasse, D-78457 Konstanz, Germany \\ ${ }^{3}$ Department of Metallurgy and Ceramics Science, Graduate School of Science and Engineering, \\ Tokyo Institute of Technology, 2-12-1-S7-14, Ookayama, Meguro-ku, Tokyo 152-8552, Japan
}

Received November 5, 2012

\begin{abstract}
Igor Yanson showed 38 years ago for the first time a point-contact measurement where he probed the energy resolved spectroscopy of the electronic scattering inside the metal. Since this first measurement, the pointcontact spectroscopy (PCS) technique improved enormously. The application of the scanning probe microscopy (SPM) techniques in the late 1980s allowed achieving contacts with a diameter of a single atom. With the introduction of the mechanically controlled break junction technique, even spectroscopy on freely suspended chains of atoms could be performed. In this paper, we briefly review the current developments of PCS and show recent experiments in advanced scanning PCS based on SPM techniques. We describe some results obtained with both needle-anvil type of point contacts and scanning tunneling microscopy (STM). We also show our first attempt to lift up with a STM a chain of single gold atoms from a $\mathrm{Au}(110)$ surface.
\end{abstract}

PACS: $07.79 . F c \quad$ Near-field scanning optical microscopes;

87.80.Ek Mechanical and micromechanical techniques;

37.10.De Atom cooling methods;

37.10.Gh Atom traps and guides;

73.23.Ad Ballistic transport.

Keywords: scanning tunneling microscopes, single-molecule techniques, atom manipulation in atomic physics, ballistic transport.

\section{Contents}



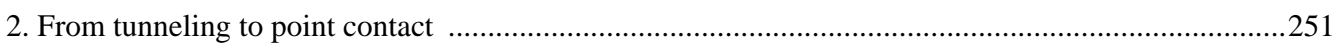

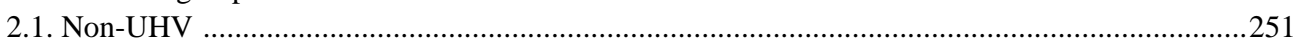

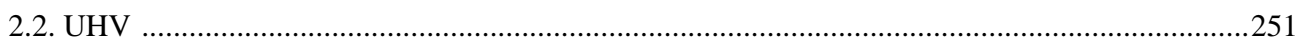

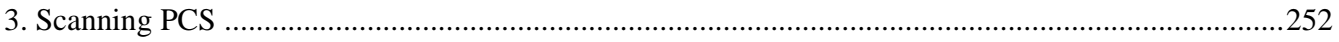

4. Conductance quantization and formation of freely suspended metallic wires ...................................253



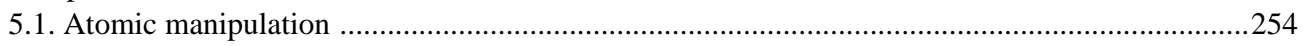

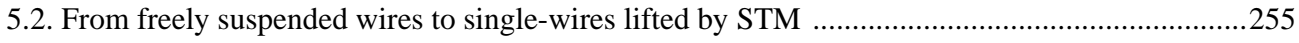

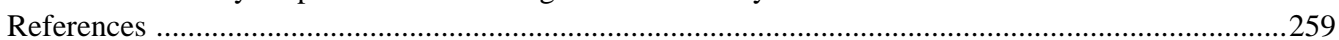




\section{Introduction}

The electrical resistance of a contact with a size smaller than the electronic mean free path is only determined by the number of quantum channels and their transmission probability through this contact. These contacts, called Sharvin or point contacts (PC), were first used to determine the electron-phonon spectral function in normal metals. Point-contact spectroscopy (PCS) developed into an ideal tool to probe a whole range of elementary excitations in all kinds of systems [1]. In recent years, PCS became an important tool to probe the order parameter in (multiband) superconducting materials like the cuprates [2] and the pnictides [3], to measure the spin polarization of magnetic materials [4] and to measure transport properties of single atoms and molecules where the local vibration modes of the atoms or molecules can be detected.

The transport through such point contacts can be characterized by different transport regimes, depending on how the diameter $d$ of the point contact scales to the elastic $\ell_{\mathrm{el}}$ and inelastic $\ell_{\text {in }}$ electron mean free path and the Fermi wavelength $\lambda_{F}$ of electrons. We can than distinguish the thermal regime, where $d \gg \ell_{\mathrm{el}}, \ell_{\mathrm{in}}, \lambda_{F}$, the diffusive regime $\ell_{\mathrm{el}} \gg d \gg \sqrt{\ell_{\mathrm{el}} \ell_{\text {in }}}, \lambda_{F}$, the ballistic regime $\ell_{\mathrm{el}}, \ell_{\text {in }} \gg d \gg \lambda_{F}$ and the quantum regime, where $\ell_{\mathrm{el}}, \ell_{\mathrm{in}}, \lambda_{F} \gg d$.

Formation of point contacts allowed observing new phenomena arising from the atomic dimensions of such junctions. Quantum transport is characterized by the fact that carriers flowing inside the constriction have discrete transverse momentum components; when the lateral dimensions of the contact are comparable with $\lambda_{F}$, only a discrete number of electronic bands are available inside the constriction which can be occupied by electrons flowing from one electrode to the other. As a consequence each band which gets occupied in the constriction contributes with a fundamental conductance quantum $G_{0}$ to the total conductance through the junction for an ideal constriction; it is commonly said that a conductance channel is open. The conductance measured in such constrictions is thus said to be quantized in units of $G_{0}$. This picture does not take into account several effects such as backscattering and reflection at the contact; as a consequence the channels are not just open or closed but are associated with a transmission probability for each channel which contributes to the total conductance. These effects are treated by the Landauer-Büttiker formalism of quantum transport [5,6].

In 1974 Yanson [7] used a metal-insulator-metal tunnel junction having a short, to measure energy resolved spectroscopy of the electronic scattering inside the metal, and could directly measure the energy dependence of the electron-phonon interaction. The introduction of the needleanvil method by Jansen et al. [8] in 1976 was a further improvement. With this technique a sharply etched metal wire, the needle, was pressed using a micrometer screw into a flat metal surface, the anvil, to form a point contact.
The contact size can be adjusted by fine-tuning the pressure applied to the tip with the micrometer screw. Transport in such point contacts is ballistic, since contacts diameters are usually much smaller than the electron mean free path. Nevertheless, diameters of such point contacts were usually much larger than the Fermi wavelength $\lambda_{F}$ of electrons, of the order of $40 \AA$ [9], so no quantization of the electron momentum occurs inside the constriction. At that time, there were already ideas of quantum transport for contact dimensions of the order of $\lambda_{F}$, but such small junctions could not be realized experimentally.

Quantum effects have been extensively studied in twodimensional electron gasses at semiconductors interfaces [10-12]. The Fermi wavelength of electrons in a semiconductor, $\lambda_{F}$, is around $40 \mathrm{~nm}$ [10], which is much larger than the Fermi wavelength in a metal (4-5 $\AA$ ). In a semiconductor, the electron transverse momentum is thus quantized more easily in the point-contact region. Measurements of the conductance as a function of the width of a contact defined by a two-dimensional electron gas of a GaAs-AlGaAs heterostructure showed quantized steps of the conductance in integer multiples of the conductance quantum unit $G_{0}$ [10-12].

In 1982, the invention of the scanning tunneling microscope (STM) by Binnig and Rohrer [13] allowed achieving for the first time atomic resolution on a well-defined surface structure [14,15]. Atomic resolution capability has become the most important feature of STM and has several significant consequences. Surface structures can be monitored at the atomic scale; this implies that the tip can be positioned with atomic accuracy on top of a well-defined atomic site on a surface. The combination of these two features leads to the ability of controlled atomic manipulation, which allows the assembly of atomic-scale devices on surfaces.

For the purpose of studying the conductance through atomic-sized point contacts a new technique has been introduced in 1992, known as the mechanically controlled break junction (MCBJ) [16-18]. The working principles of this technique rely on bending a notched wire mounted on a bending beam in a three-point bending configuration. The substrate is bent by a piezoelectric element and it allows the wire to break at the notch site. Two clean metal surfaces with atomic dimensions facing each other can thus be obtained. These surfaces can be brought in or out of contact in a very controlled manner. The high mechanical stability of a MCBJ has been found to be particularly suited for the investigation of conductance quantization on metallic point contacts; several MCBJ experiments on atomic point contacts of different metals showed distinct steps in the conductance behavior $[16,18,19]$.

In the last 20 years also the development of STM, at or below liquid helium temperatures improved enormously [20]. One of the biggest problems in the design of lowtemperature STMs was the piezoelectric positioning sys- 
tem, that often fails to work at these low temperatures. The Beetle and the Pan-type walker coarse positioner solved this problem and are now widely used in low temperature STMs. Nowadays, one can even buy commercial piezo positioners, that reliably operate at these temperatures and at high magnetic fields [21].

A next step would be to combine the possibilities of the STM and the point-contact spectroscopy technique. From the point-contact spectroscopy perspective, it would be interesting to map the elementary excitations, like the local order parameter of superconducting materials, or to map the variation of the polarization. From a STM perspective, it would be interesting to study the transport of artificial structures that are created and manipulated by STM.

In this paper, we briefly review the current developments of point-contact spectroscopy and show recent experiments in advanced scanning point-contact spectroscopy. We start with a comparison of point contacts made with the traditional non-UHV (ultra high vacuum) needle anvil technique and UHV STM based point-contact spectroscopy. Thereafter, we describe our Leiden efforts to do both STM and needle-anvil type of point contacts.

Afterwards we switch to UHV-STM based point-contact spectroscopy and give an overview of the possibilities of creating freely suspended chains of atoms in a MCBJ and manipulate atoms by STM. Finally, we show the prospectives of what is possible with a STM, with the assembly and manipulation of single gold chains and discuss the possibility to lift gold atomic wires and do spectroscopy on them.

\section{From tunneling to point contact}

The formation of a point contact is characterized by an abrupt change in the transport regime through the constriction. Before the point contact is created electrons tunnel through the barrier between the metal electrodes; when a point contact is made, electrons can flow through the conduction channels open in the junction and transport occurs in ballistic regime. The environment influences dramatically how the point contact is made. We can distinguish two scenarios: a non-UHV and a UHV environment.

\subsection{Non-UHV}

In a non-UHV environment, a very thin native oxide layer, plus adsorbates of organic materials, are always present on both the tip and sample. A very important requirement is to make a clean metallic contact between the sample and the tip. For this purpose the tip is moved slowly with a micrometer screw or a piezopositioner to the sample, and when both parts are firmly pressed together, the greatest stress occurs at the point where the needle touches the sample. Here the oxide layer breaks and forms a small direct metallic contact between the two parts: the point contact. Note that the presence of the oxide layer is not only a disadvantage: it can be helpful because it reduces the conducting contact area between the tip and sample, while the mechanical contact area is much larger so that stable contact sizes between 4 and $100 \mathrm{~nm}$ can be made.

\section{2. $U H V$}

In a UHV environment, the native oxide layer and adsorbates of organic materials can be removed during the sample preparation stage by sputtering-annealing cycles, or in a MCBJ by breaking the wire in cryogenic vacuum.

The experimental study of quantum transport requires a highly controlled way of creating and breaking the contact between metallic leads. Junctions which dimensions are down to the atomic scale are made and controlled usually by STM [9,22-28] or by conductive AFM [29,30].

With STM, very narrow constrictions can be created by bringing the tip close to the surface until contact is achieved. In STM the distance between tip and surface can be controlled down to the pm scale, so atomic point contacts can be formed with high accuracy.

The character of electron transport has been found to change from tunneling through the vacuum barrier to quantum transport while bringing an STM tip in contact with the surface $[22,31-35]$. The passage from one regime to another is usually not a smooth transition: abrupt jumps in the current recorded across the constriction or in the resistance of the point contact are observed when the tip is brought very close to the surface [31,32]. The resistance of the constriction drops suddenly to a value close to $R=12.9 \mathrm{k} \Omega$; this value corresponds to a conductance through the constriction of the order of $1 G_{0}$ which is associated with a single-atom contact. The drop in the resistance, or the jump of the current, observed while decreasing the tip-sample distance marks the passage from tunneling to contact regime. An example of drop of the tunnel resistance while moving the tip towards the surface is shown in Fig. 1 in which an Ir tip is brought in contact with an Ir surface [32].

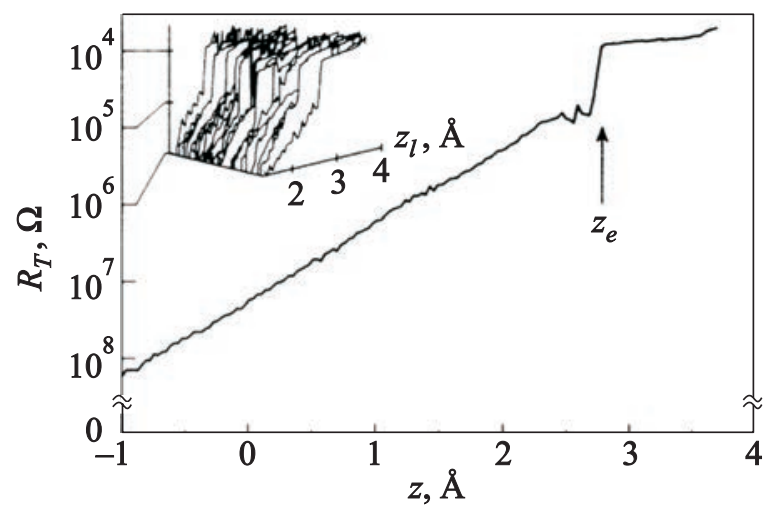

Fig. 1. Tunnel resistance as a function of an Ir tip excursion $Z$ towards an Ir surface. Positive $z$ corresponds to decreasing gap width. Transition to point contact is shown by an arrow for a tip excursion $z_{e}$. The inset represents the degree of reproducibility of the transition. Image reprinted with permission from [32]. 
Mechanical properties of atomic-sized contacts have been investigated theoretically by molecular dynamics computer simulations and first-principle calculations [36-42]. The sudden jump-to-contact can be explained by taking into account the balance of forces involved inside the constriction [36,40,42]. At very small distances between tip and sample the apex atoms of the tip, as well as the underlying atoms on the surface, experience two competing forces [40]. The first one is the strong metallic attractive force between the apex atom of the tip and the underlying metal atoms of the surface, occurring when two metals are very close to each other [36,37]. This force pushes the apex atom of the tip towards the surface and vice versa. The competing force is a spring restoring force exerted by the tip lattice, which keeps the apex atoms at their unperturbed positions inside the tip structure. The balance between these two forces gives rise to a double well potential inside the constriction [36,40,41]. When tip and sample are still far from each other the two minima of this potential are well separated; this means that the restoring spring force is stronger thus keeping the tip apex atoms bound to the tip structure. While decreasing the tip-sample distance the metallic adhesive force between tip and sample becomes stronger than the restoring spring force; to minimize their energy the atoms of the tip relax towards a new potential minimum and the tip is thus abruptly brought in contact with the sample [38,39,42]. Transport abruptly changes from tunneling to contact regime. A sudden decrease of the actual distance between tip and sample has been observed while rigidly approaching one to the other [36,38,39]; this drop has been found to occur at the same rigid tip-sample distance in which a jump in the conductance was observed [38]. The understanding of the phenomena arising from the reduced dimensions of point contacts in STM allowed many improvements in the field of atomic-dimensions electronics, and it opened the doors to new applications of the STM in surface science.

\section{Scanning PCS}

As mentioned before, point contacts were first employed to study phonon densities of states in normal metals. PCS has been widely employed to study the phonon emission effect in metals by deriving the electron-phonon spectral function from transport measurements. The measurements with the traditional micrometer-controlled needle anvil technique are often limited to a single position on the sample due to a lack of the ability to move the sample in a controllable way.

A big improvement in PCS would be the ability to map the PCS data on the surface by moving the sample in a controllable way. In Leiden, we started to explore the possibility to do scanning PCS. For this purpose, we built a scanning PCS system, where we used a modified STMinsert [43] with an Attocube ANPz101 z-direction nano- positioner and a scantube to move the tip in the $x$ and $y$ directions. For the first tests, only the $z$ direction was used. As a comparison, also point contacts were made with an insert where the tip was connected via a drive shaft to a micrometer screw mechanism, which is capable of moving the tip with $1 \mu \mathrm{m}$ per revolution.

As a test of the system, the well-known phonon spectrum of copper-copper point contact was measured. The $125 \mu \mathrm{m} \mathrm{Cu}$ tip was electrochemically etched in $25 \% \mathrm{HCl}$ solution [44] and had a typical apex of $10 \mu \mathrm{m}$ as can be seen in Fig. 2(a). The sample was a thin oxygen free copper foil, which was etched in $25 \% \mathrm{HCl}$ solution until it looked shiny. The point-contact measurements were performed at $4.2 \mathrm{~K}$ in a liquid helium bath cryostat. The conductance and the phonon spectrum were measured using standard lock-in technique.

The point contact was made by carefully moving the tip with the piezo positioner or micrometer screw while simultaneously measuring the resistance between the tip and the sample. Before the contact was formed, a high constant resistance was observed, which is the result of combination of the native oxide and the vacuum gap between the tip and sample. When making the contact, the tip will first approach the oxide covered surface layer of the sample. When the tip touches the sample, it needs also to break the thin oxide layer to form a metallic contact. Unfortunately, we cannot distinguish these processes during the approach.

We noticed, instead, that if we made our first contacts with a fresh tip and fresh sample, when we made a metallic contact and retracted the tip just far enough that we lose the metallic contact, the next approach takes much longer than expected from the retraction distance. If the crack in the oxide layer forms at a different place than before, the piezopositioner needs a lot of steps to break the oxide layer. It is also possible that instead of breaking the oxide layer, a metallic contact is formed by deforming the tip as can be seen in Fig. 2(b), where the tip is imaged just after good ballistic contacts were measured.

The main drawback of the low-temperature piezopositioners is that they are designed for accurate positioning (several tens of nm), but not to apply forces large enough
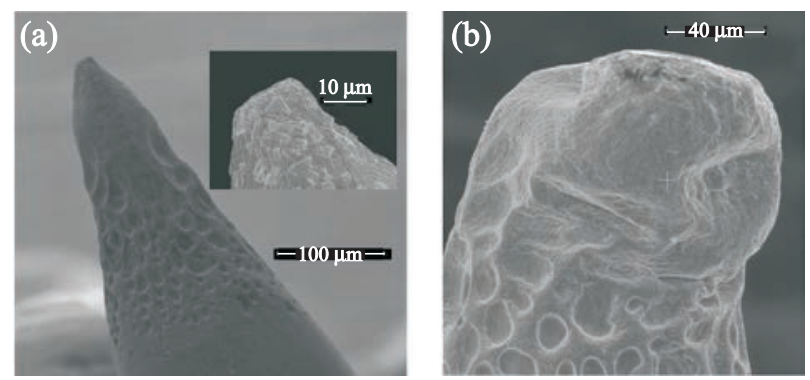

Fig. 2. A scanning electron microscopy image of two different electrochemically etched Cu-tip before (a) and after (b) a pointcontact spectroscopy measurement with an piezopositioner. 
to break oxide layers. In contrast, the micrometer screw can continuously apply the same force and break the oxide layer in a continuous way.

Atomic point contacts can, instead, be made under significantly stable conditions using either scanning probe microscopes or mechanically controlled break-junctions. These techniques allow creating point contacts for which the cross-section is made of just a few atoms by bringing the metal electrodes towards each other. No force is needed to form the point contact, on the contrary, atomic point contacts form spontaneously when the distance between the electrodes is lower than a threshold distance. Techniques employed to create such small contacts can control the distance between the electrodes in a very precise way so stability of such point contacts is usually ensured.

\section{Conductance quantization and formation of freely suspended metallic wires}

In STM and MCBJ the high stability and the fine control of the distance between the metal electrodes allowed observing new phenomena occuring in the contact region while forming atomic point contacts. An important example is the formation of metallic atomic chains between the electrodes when these are moved apart after being in contact. Metal atomic chain assembly and breaking is done by bringing two metallic electrodes in contact between them and then separating them back; during this manipulation the conductance across the junction is measured.

Transport measurements through freely suspended atomic wires require measuring the total conductance over the junction. Each conductance curve depends on the actual atoms configuration inside the constriction, so curves recorded in identical conditions can be very different from each other. Therefore statistical measurements and analysis are employed to characterize transport properties in such junctions. This analysis is done by building conductance histograms from a large number of individual conductance curves, in which the most probable conductance values occurring through a constriction are distinctly highlighted $[19,23,25,27,28]$.

The signature of quantum effects arising from the atomic-dimensions of such point contacts is clearly shown, at least for simple, monovalent metals, by the fact that, while making or breaking atomic-size contacts, the conductance through the constriction takes preferably values equal to integer multiples of the quantum conductance unit $G_{0}$ $[19,23,25]$. Moreover, while stretching the contact the conductance of an atomic-size constriction decreases in steps which amplitude is of the order of the fundamental quantum conductance $G_{0}$. The same behavior is observed in reverse direction when the contact between the electrodes is established again after breaking it [23,25-27,30,45]. During the manipulation several atomic rearrangements take place inside the constriction, thus changing the contact diameter at the atomic scale. These atomic rearrangements have been found to lead to the step-like behavior of the conductance through the junction [45]. By means of simultaneous measurements of force and conductance distinct jumps in the force behavior have been observed where conductance steps occur [29]. The number of atoms and the valence of the atoms which are part of the contact determine the number of transmission channels inside the constriction and thus the value of the conductance in terms of the conductance quantum unit $G_{0}$. This is a rough approximation and the actual value of the conductance steps is related to the number of conducting modes and to the transmission function of each transmitting mode [5,6]. The valence of the metal atoms which compose the junction thus influences the amplitude of the measured conductance steps [22,28].

The formation of a metallic atomic wire has been observed experimentally when the contact between the two electrodes has a cross-section of just one atom. For Au, in this configuration, the total conductance of the constriction is about one quantum unit of conductance; the conductance trace shows a plateau at a level of $1 G_{0}$. This plateau has a length of several $\AA$; that means that two electrodes which are in contact by one atom stay in contact while stretching the junction for several $\AA$ before breaking (see Fig. 3).

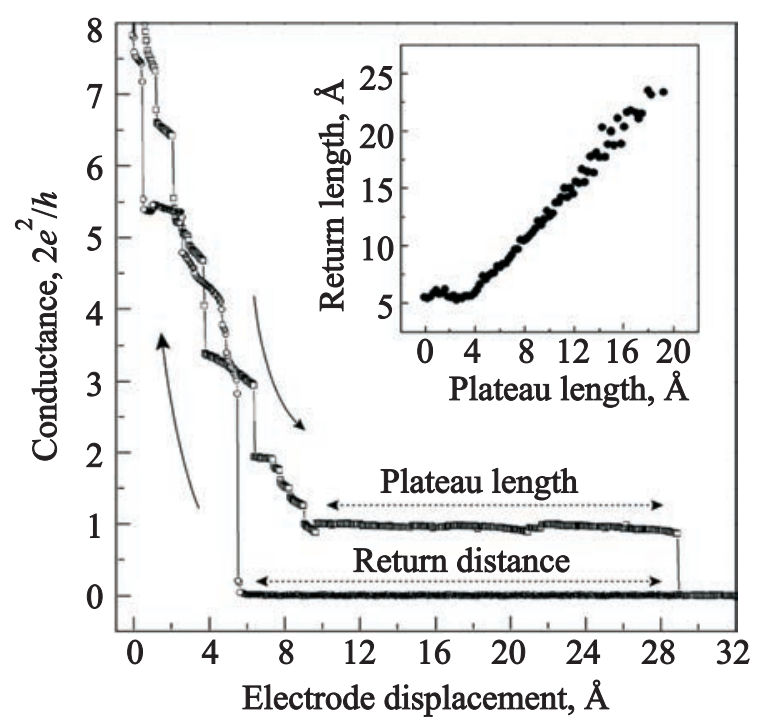

Fig. 3. Conductance as a function of the displacement of two gold electrodes with respect to each other in an MCBJ experiment at $4.2 \mathrm{~K}$. Open squares represent the conductance trace while breaking the contact; a long plateau with a conductance near $1 G_{0}$ is observed before the contact breaks. Open circles show instead the conductance trace while forming back the contact after breaking it; the electrodes need to displace towards each other by a slightly larger displacement than the one in which the electrodes stay in contact while opening the junction. The inset shows the return distance as a function of the length of the long plateau; the $5 \AA$ offset is probably due to the elasticity of the atomic structure. Image reprinted with permission from [25]. 
Because of forces balancing inside the constriction $[30,45]$ atoms in the bulk electrodes relax inside the constriction to minimize their total energy; this mechanism leads to the assembly of a metallic chain of individual atoms between the electrodes [22,25,27,30]. The mechanisms involved in the formation of chains by making or breaking atomic-size contacts have also been investigated theoretically by molecular dynamics simulations [28,30,46,47]. It has been pointed out that metallic chains form just before the contact breaks because of elastic deformation stages of the constriction due to atoms mechanical rearrangements $[28,30]$. It has moreover been observed that for some metals ( $\mathrm{Au}$ and $\mathrm{Pt}$ ) the bonds in low-coordinated structures such as chains are very strong relative to bulk bonds [28]. Not all metals can form single-atom chains: the phenomenon is related to the valence of the metal under study and for pure metals it is limited to the heavy elements at the end of the $5 d$ series, Ir, Pt and Au $[22,28]$. Recently it has been observed that atomic chain formation during stretching of atomic-size contacts is strongly enhanced by oxygen incorporation [48]. The presence of oxygen has been found to allow freely suspended wires formation even in metals that usually don't form such structures.

The high stability and reproducibility in creating atomic point contacts with scanning probe microscopy techniques has also other important applications; it has been found that single adatoms lying on a metal surface can be displaced and manipulated on the surface using the tip electrode of the microscope as element to contact and move them. Single-atom manipulation has been possible because of a growing understanding of mechanical processes occurring at the atomic scale while forming an atomic-size point contact.

\section{Manipulation}

\subsection{Atomic manipulation}

Recently advances in scanning tunneling microscopy allowed manipulating matter at the atomic scale. With STM a high control of the position of the tip towards the surface can be achieved; atomic point contacts can be made in a highly reproducible way. As a consequence single atoms can be positioned with atomic accuracy on top of a well-defined site of a surface. Manipulating single atoms on a surface involves a series of steps which result in a selective making and breaking of chemical bonds between atoms. The manipulation can be done either vertically or horizontally with respect to the surface orientation [49]. In parallel or horizontal processes an adsorbed atom or molecule is induced to move along the surface, while in perpendicular or vertical processes the atom or molecule is transferred from the surface to the tip or vice versa. When an STM tip gets in contact with a metallic surface strong interactions occur between the two electrodes. In a few experiments it has been observed by scanning the surface after contact that a single or a few atoms of the tip have been deposited on the surface, or that an atom of the surface has been picked up by the tip upon retraction $[31,35,50]$. These processes take the name of perpendicular or vertical manipulation processes. An atom or molecule lying on a surface binds to the tip while being in contact with it; in this configuration the energy barrier between tip and sample has collapsed; the strong interaction between tip and sample is the main driving force for atom transfer $[49,50]$. It has been observed that vertical manipulation can be improved by applying a voltage pulse over the junction at small tip-sample separations [35,49,51-53]. This manipulation takes the name of field-evaporation process; voltage pulses are necessary to make the adsorbate overcome the energy barrier between tip and sample. One of the first vertical manipulation processes is the deposition of ordered arrays of Au mounds on top of an Au(111) surface [52] in which mounds with diameters down to $100 \AA$ were formed by the application of $600 \mathrm{~ns}$ pulses of $+3.6 \mathrm{~V}$ to the gold sample. Vertical processes have been employed in more recent works to build atomic switches with an STM [51,53]. In Ref. 53 a single Xe atom is moved in a reversible way from two stable positions, respectively, on the STM tip and on a Ni(110) surface. The state of the switch changes when the atom is transferred from the tip to the surface and vice versa; the switching is set by the application of a voltage pulse across the junction. A highconductance state is found when the atom is bound to the tip; this state is reached by applying a positive voltage pulse of $+0.8 \mathrm{~V}$ to the tip. A low-conductance state is found instead when the atom is bound to the surface; this state is reached by applying a negative pulse of the same amplitude. In this way it has been possible to transfer a Xe atom on a well-defined site of the surface in a reproducible way. When the pulse is applied over the junction an atomic point contact is formed. This can be confirmed either by imaging the surface after the application of the pulse or by measuring the conductance through the junction while applying the pulse. Conductance values have been found to lay around $1 G_{0}$, confirming the fact that a single-atom contact has been established between tip and sample [50,51].

Horizontal processes involve lateral manipulation, in which adsorbates lying on the surface are dragged by the tip from a starting position to a final position on the surface. The strong interaction occurring between the tip and an adsorbate at small distances creates a potential well located under the tip, which traps the adsorbate [19,54,55]. This interaction leads to the formation of a chemical bond between the STM tip and the adsorbate. The latter can then move from one binding site to another on the surface by moving the tip at constant height over the surface. During the time of the manipulation the tip and the adsorbate are in contact. The first experiment in which single atoms are dragged over the surface by the tip is given in Ref. 56, in 
which single Xe atoms are positioned on a nickel surface with atomic precision. The manipulation is done by bringing the tip in contact with an adatom, previously located by scanning the surface. The tip moves at constant height mode over the surface; the adatom is displaced by the tip movement to a different site of the surface. Afterwards the tip is retracted thus leaving the adatom at the final position on the surface. There are several examples of experiments in which STM is employed to manipulate single-atoms on a surface [54,56-59]. The 3D STM images in Fig. 4(a) and (b) show an example of single-atom manipulation performed in STM at $6 \mathrm{~K}$, in which $36 \mathrm{Ag}$ adatoms are displaced on top of a $\mathrm{Ag}(111)$ surface to form a quantum corral [57].

The manipulation is characterized by a threshold tip height; above this height the interaction between tip and sample is too weak to allow atom manipulation. One way of monitoring the tip height is by setting a high threshold tunneling current while approaching the tip towards the surface; common current values are of the order of 10-50 nA $[53,58]$, leading to the tip being in contact with the adatom. The threshold height can also be controlled by recording the resistance, or analogously the conductance, across the junction while lowering the tip position over the surface [50,57]. A drop in the resistance to a value of the order of

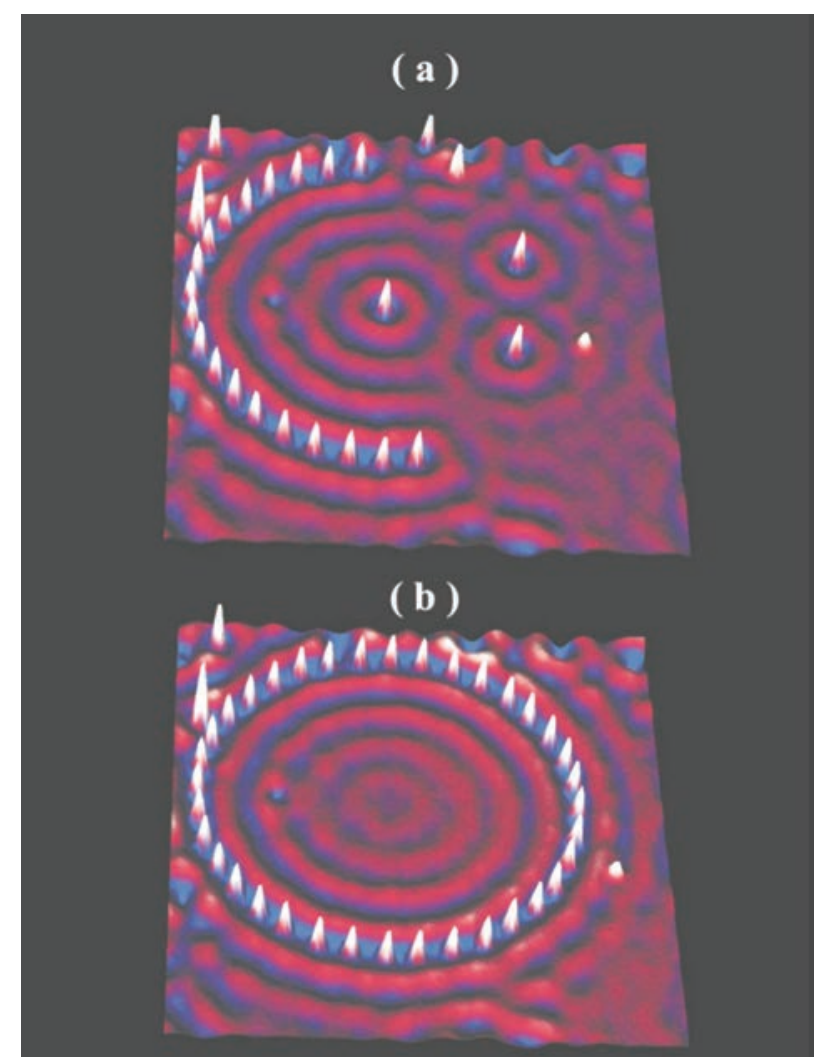

Fig. 4. Construction of a quantum corral by STM manipulation at $6 \mathrm{~K}$. (a) 3D STM image taken during the construction of the corral. (b) 3D STM image after completion of the corral. White protrusions represent the $36 \mathrm{Ag}$ adatoms which form the quantum corral (diameter: $31.2 \mathrm{~nm}$ ). Image reprinted with permission from [57]. $\mathrm{k} \Omega$, as well as a jump in conductance to values around $G_{0}$, is the signature that the adatom lying on a metal surface is in contact with the STM tip.

The possibility to manipulate single-atoms on a surface gave the opportunity to scan metal surfaces while keeping the tip in contact with a metal adatom [54,60-62]. This technique can be seen as a new insight into surface characterization and it takes the name of quantum point-contact microscopy (QPCM) [61]. In QPCM the conductance over the junction is recorded while the tip scans the surface while being in contact with an adatom; during scanning the tip displaces laterally the adatom on the surface. QPCM images represent conductance maps of the scanned surfaces; conductance values during scanning are of the order of $G_{0}$, indicating that electronic transport occurs through a single atom contact. As a consequence the technique has several advantages with respect to normal STM imaging. An example is a higher stability of the imaging process, due to the fact that the tip is in contact with the adatom so atoms in the tip structure and on the surface are in a relaxed configuration; conductance fluctuations can thus be avoided and atomic resolution is easily achieved $[60,61,63]$. Moreover the technique has a strong chemical sensitivity of the local environment of the adatom [59,61,62]. Chemical sensitivity allows distinguishing between different surface sites; hcp and fcc sites on an $\mathrm{Au}(111)$ surface have been found to have different conductance values in QPCM images [61]. It's consequently possible to identify adatoms' favorite adsorption sites on the surface as the sites having higher conductance values. Quantum point-contact microscopy is thus suited for studying quantum transport through atomic-sized point contacts as well as characterizing chemical and structural properties of a metallic surface.

\subsection{From freely suspended wires to single-wires lifted by STM}

In the last decade extensive studies on atom manipulation and transport through atomic-size point contacts in STM allowed understanding and controlling the dynamics involved in such processes. This dynamics is nowadays exploited to build and manipulate atomic-size devices on surfaces. A few examples are the assembly of atomic-size structures on a surface $[57,58,64-66]$ or the manipulation of mesoscopic systems such as organic molecules [67-73]. The high stability and reproducibility of STM in manipulating adsorbates deposited on a surface is of fundamental importance to understand transport characteristics in view of future applications in functional nanoscale devices. A few steps have already been accomplished; recently single $\mathrm{Au}$ atoms have been assembled on top of a $\mathrm{NiAl}(110)$ surface to form single-atom chains [64-66]. Single Au atoms are laterally manipulated with an STM to form linear Au chains up to 20 atoms long [64,65] (see Fig. 5). 

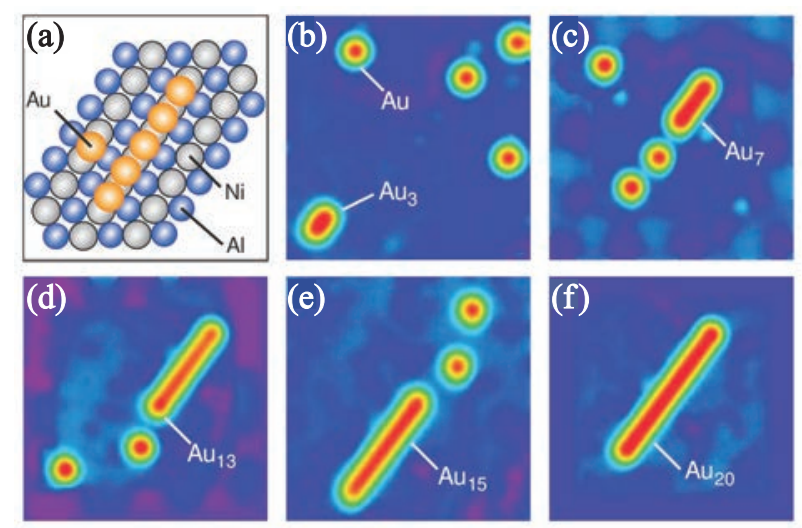

Fig. 5. (a) Structure model of a Au chain construction on top of a $\mathrm{NiAl}(110)$ surface. Au atoms bind preferably on top of bridge sites between $\mathrm{Ni}$ atoms. (b) to (f) STM constant current images ( $I=1 \mathrm{nA} ; V_{\text {sample }}=2.1 \mathrm{~V}$ ) showing different steps of the construction of a gold chain up to 20 atoms long $\left(\mathrm{Au}_{20}\right)$. Image size is $95 \AA$ by $95 \AA$. Image reprinted with permission from [66].

The chains are assembled along the [001] direction of the NiAl substrate. Each Au atom binds on top of a bridge site between two Ni atoms. The spacing between the gold atoms is thus the same as the one between $\mathrm{Ni}$ atoms along the [001] direction; this one (2.89 $\AA$ ) is comparable to the separation of bulk Au atoms (2.88 $\AA$ ). The $\operatorname{NiAl}(110)$ surface works thus as a natural template for the assembly of gold chains. Similar experiments have been performed a few years later using an insulating film deposited on a metallic surface as substrate to deposit single gold atoms $[64,74,75]$. In order to assemble chains consisting in single nanoscale devices atoms in the chain have to be strongly bound to each other, their electronic properties have to reflect the one-dimensional nature of the built structure. The spacing between the manipulated atoms in the chains should be thus comparable to the bulk spacing so that a one-dimensional band structure develops from a singleatomic orbital [66]. For this purpose a simple solution could be to assemble single-atom gold chains on top of a clean $\mathrm{Au}(111)$ surface. Using the same material for the substrate and the adatoms preserves the spacing between atoms in the assembled chains; moreover (111) metal surfaces have close-packed lattices so a single-atom chain lying on a (111) surface "feels" like an isolated system thus developing stronger bonds between the chains atoms than in the bulk material [76]. Another good candidate as substrate for lifting single-atom chains is a reconstructed (110) surface, in which the missing row reconstruction (MRR) leads to the formation of a pattern of single-atoms chains separated by $8.16 \AA$ from each other and oriented along the [110] direction [77].

For the purpose of building nanoscale devices by STM, the assembly of such atomic wires should be accompanied by a further step which consists in lifting and isolating these wires upon tip manipulation. This requires breaking the



Fig. 6. (Color online) The $\mathrm{Au}(110)$ surface with missing raw reconstruction (MRR). In different colors are shown the layers of chains, where the top one can be contacted and pulled with a STM tip.

bonds between the chains' atoms and the metal atoms of the surface underneath while keeping the atoms of the chain bound to each other. The process has to take into account the balance between several forces occurring between the chains' atoms, the metal surface and the tip apex atoms. The bond strengths between single Au atoms of a chain can be probed by measuring the force required to add or remove an atom from the chain. This has been found to be in the order of $10-100 \mathrm{pN}$ for a $\mathrm{NiAl}(110)$ substrate [65]. Moreover gold atoms have been found to form stronger bonds in low-coordinated structures than in bulk structures [76]. The dynamics involved in this process is not very different from the one involved while forming freely suspended wires between electrodes. The tip gets in contact with the first atom of a chain; contact is monitored by measuring either the conductance or the resistance across the junction; single atoms of the chain have to relax between the tip and the surface one after the other while retracting the tip from the surface so that the entire chain would be "spontaneously" lifted by means of tip retraction. Since adatoms are likely to adsorb on bridge $[65,66]$ or hollow sites of the surface $[54,55,60,61]$ several bonds have to be broken between the chain and the underlying surface while vertically lifting the wire. The tip has then to follow a specific trajectory while retracting in order to break one bond at the time; this trajectory is defined by two tilting angles $\alpha$ and $\beta$.

On the $\mathrm{Au}(110)$ surface with MRR one can approach the pulling problem as follows. An edge atom in the top row of the MRR has six nearest neighbors, one next to it in the row, four in the layer below and another one directly beneath it.

Most promising for the chain formation is the tip motion in a direction in which the bonds of the edge atom towards its nearest neighbors in the bulk are broken one after the other (1, 2, 3, 4 in Fig. 7(c)). In order to achieve this, an initial symmetry in tip motion needs to be broken.

As schematically sketched in Fig. 7(a) one can define a normal plane ( $n$ in Fig. 8), that is spanned by the vector 


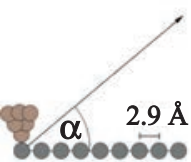

(a)

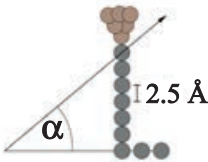

(b)

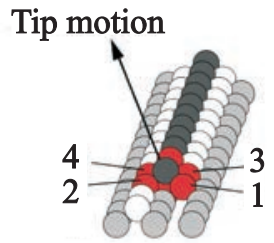

(c)
Fig. 7. (Color online) Explanatory drawings for pulling angle $\alpha$ and tilting angle $\beta$; (a) tip (brown) in single-atomic contact with the atom at the end of the chain (grey); (b) freely suspended oneatomic gold chain after tip motion under an angle $\alpha$ in respect to the chain; (c) single ending chain of MRR; the target atom (grey) is surrounded by its five nearest neighbors (red); direction of tip motion indicates different distances between the tip position and the individual nearest neighbors resulting in breaking of bonds between target atom and nearest neighbors one after the other.

normal to the surface and the one along the MRR. Pulling along a direction in this plane seems less favorable. In this case, at least two bonds would have to be broken at the same time (e.g., $(1,2)$; $(3,4)$ in Fig. $7(\mathrm{c}))$. In order to break this initial symmetry given by the atomic configuration the tip needs to move in a direction leading out of this mirror plane. The direction of the chains on the surface and the direction of this not yet defined tip motion defines a plane called $t$ (Fig. 8), which is tilted with respect to the mirror plane by a tilting angle $\beta$. The boundary values are the previously excluded case $\left(\beta=0^{\circ}\right)$ and the movement along the crystal surface in contact $\left(\beta=90^{\circ}\right)$. By tip motions in the direction defined by $\alpha$ and $\beta$, the bonds of the uttermost atom in the chain towards its nearest neighbors in the bulk could be broken one after the other (1, 2, 3, 4 in Fig. 7(c)).

The pulling direction is thus not just along the vertical $z$ axis but it includes components along $x$ and $y$ axes; this

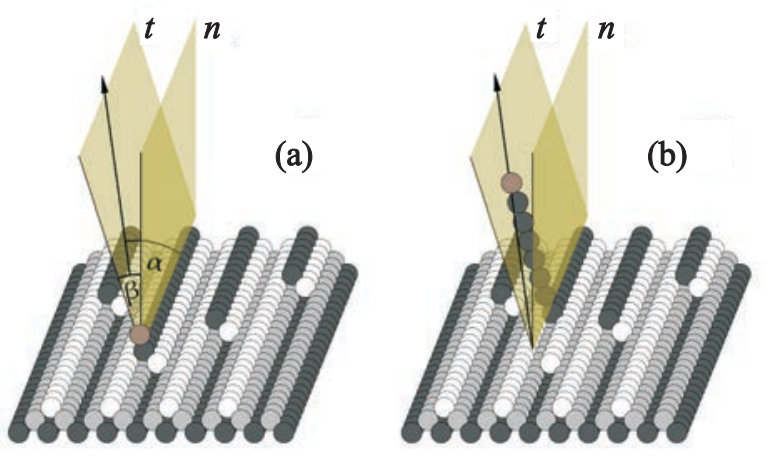

Fig. 8. (Color online) Illustration of chain pulling idea on the $\mathrm{Au}(110)$ single crystal (different gold layers color coded); pulling direction in dependence of pulling angle $\alpha$ and tilting angle $\beta$; (a) tip apex atom (brown) establishing contact with target atom on the end of the chain; definition of pulling direction $(\alpha, \beta)$ indicated by pointed direction (b) freely suspended monoatomic gold chain between tip and crystal after having been pulled off from the surface by tip retraction.

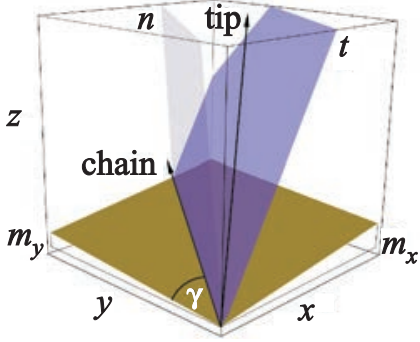

(a)



(b)
Fig. 9. (Color online) Drawing of an arbitrarily oriented surface in 3D (a) and STM image showing the exemplary chain direction (b). (a) By $m_{x}$ and by $m_{y}$ the orientation of the simplified in 3D is defined. The direction of a single chain on the surface and the direction of the tip motion are indicated. The angle $\gamma$ defines the direction of the chains in function of $x$ and $y$. (b) STM image at $300 \mathrm{mK}$. The missing row reconstruction is displayed and by the orientation of the rows the chain direction $\gamma$ is defined.

process requires controlling the tip motion in a three-dimensional trajectory.

By looking at an acquired STM image the chain direction $\gamma$ can be determined (Fig. 9(b)). It defines the orientation of the parallel rows relative to the $x$ and $y$ direction of the coordinate system for control of the piezoelectric actuators. The surface that these chains lie on, theoretically treated as an ideal plane, is in general not perpendicular to the cartesian [001] direction. So slopes in $x$ and $y$ direction, $m_{x}$ and $m_{y}$, respectively, need to be determined to define the surface orientation in 3D. They can also be obtained from the acquired STM image. The orientation of the chains in 3D is thus defined in its most general form by $m_{x}, m_{y}$ and $\gamma$. In the experiment first the surface orientation $\left(m_{x}, m_{y}\right)$ and the chain direction $(\gamma)$ are determined from an initial scan. Then the tip motion $(\alpha, \beta)$ can be defined.

After imaging in STM mode, the STM feedback is "frozen", the quantum point contact is established by moving the $z$ piezo towards the surface while monitoring the conductance. After establishing the contact the tip motion along the predefined angle can be controlled by moving (with a Labview program) the $X, Y$ and $Z$ piezomotors of the STM.

Preliminary STM measurements with the above described method were performed at $300 \mathrm{mK}$ in UHV on $\mathrm{Au}(110)$ with MRR and a Pt/Ir tip. Chain formation was not observed consistently. The longest conductance plateau that was measured was $4 \AA$ (Fig. 10). Also no missing atom or chain was found after pulling off experiments, but rather re-deposition of tip material on the surface where the tip made contact to the sample (insets I and II of Fig. 10). This could be either PtIr atoms or gold atoms picked up during STM imaging scans prior tip-sample contact.

One way to reduce the coupling strength between the adatoms and the underlying surface is to grow a thin insulating film on top of the metal surface before adatoms deposition. Similar experiments to the one described in Ref. 65 


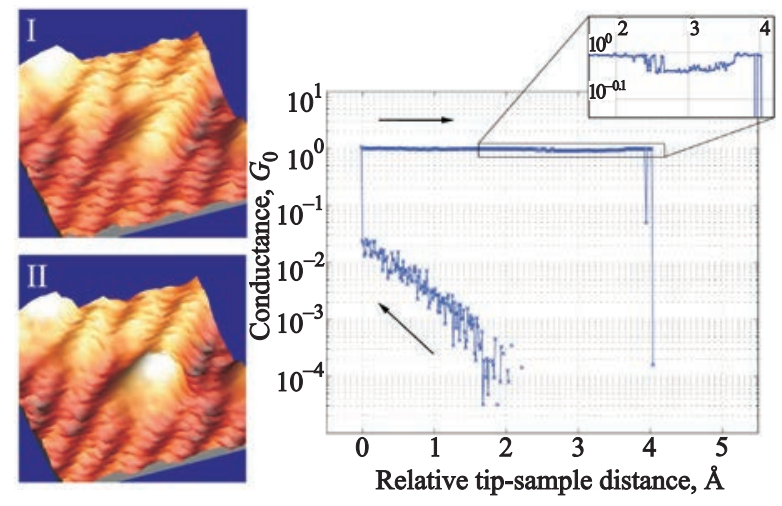

Fig. 10. Conductance trace showing a $4 \AA$ conductance plateau at $1 G_{0}$. Atomic rearrangement in the junction is observable in the plateau as well as a jump out of contact and a jump into contact before the final breaking. The STM images on the left show the deposition of tip material in the center, the spot where tip and sample touched.

have been performed a few years later using a thin ( $5 \AA$ ) insulating film deposited on a metallic surface as substrate to deposit single gold atoms $[64,74,75]$. It has been observed that one-dimensional chains assemble spontaneously along the [001] direction of a thin alumina film deposited on a $\mathrm{NiAl}(110)$ surface; single-atom chains have been identified by imaging the surface after atom deposition [64,74]. A very similar phenomenon has been observed on top of an ultrathin $\mathrm{MgO}$ film deposited onto a $\mathrm{Ag}(001)$ surface [75]. Lifting single-atom chains requires an extremely accurate control of the manipulation process and also a significantly high stability of the system. For this purpose STM operating at He temperature $(4 \mathrm{~K})$ are usually employed for atom manipulation in order to insure a high stability of the system during the process. Larger systems such as organic molecules are easier to manipulate by STM than single atoms [68-70,72,73]. Stability is also enhanced by the fact that systems as organic molecules often include a couple of ligands moieties which allow the molecules to bind covalently to the metal surface onto which they are deposited [68,72,73]. STM manipulation has been performed on a very wide range of organic systems, from very small binary molecules [70] to more complex systems [68,69,72,73] which include large selfassembled molecular wires [67,71].

In Refs. 68, 72, and 73 single molecular wires consisting in PTCDA (perylene-3,4,9,10-tetracarboxylic dianhydride) molecules are lifted from a $\mathrm{Ag}(111)$ surface with an STM tip. The tip is brought in contact with one of the ligand moieties of the molecule in order to establish a strong covalent bond with the molecule before lifting it up. During the approach the current curve is recorded across the junction versus the tip excursion towards the surface [72]. A jump of the current is observed when the contact between tip and molecule is established; in the same way a current drop is observed when the contact is broken. After contacting the tip the molecule is lifted upon tip retraction; this manipulation allows studying the molecule transport properties while changing its structural configuration inside the molecular junction. The molecule stays in the junction during the whole lifting process; the conductance measured across the molecular junction remains larger than usual tunneling contributions for retraction lengths comparable to the linear length of the molecule, showing that the molecule has been completely lifted from the horizontal position to the vertical position [68]. In the horizontal configuration the molecule lies on the surface while the vertical position corresponds to the configuration in which the molecule is bound from one side to the STM tip and from the opposite side to the underlying surface via the ligands moieties.

Recently an experiment in which very long self-assembled molecular wires are lifted by an STM tip has been performed [67,71]. In this experiment long one-dimensional molecular chains form spontaneously on top of heated $\mathrm{Au}(111)$ surface [67]. Such wires are first identified by STM imaging. The tip is then positioned at one end of the chain and brought in contact with it in order to establish the electronic contact (see Fig. 11). To lift the chain up the tip is retracted vertically until the wire is completely removed from the underlying surface while staying bound to the tip. Scanning the surface after manipulation confirms that the chain has been completely lifted from the surface by the tip. STM images taken after different manipulation steps are represented in Fig. 11. These molecular wires are extremely long compared to atomic wires; their length can

(a)

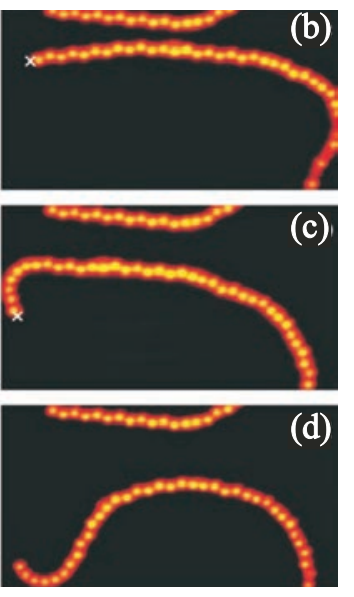

Fig. 11. (a) Schematic representation of lifting a single molecular chain with an STM tip. After contacting the tip with the molecular chain this one can be lifted upon tip vertical retraction because of its flexibility and weak interaction with the substrate. (b) to (d) STM constant current images of the same area after different vertical manipulation steps (images sizes: $25.4 \mathrm{~nm}$ by $13.7 \mathrm{~nm}$ ). The cross indicates the position of the tip during approach and retraction. The manipulated chain changes its shape during the pulling processes. The visible top chain serves as reference. Image reprinted with permission from [67]. 
reach even $20 \mathrm{~nm}$ [67]. Understanding both the dynamics involved in the lifting process and the intrinsic intramolecular transport properties of such junctions is of primary importance in view of future applications in nanoscale and molecular electronics, in which such wires could be used as building blocks for nanoscale devices. Moreover the characterization of such junctions contributes to the general understanding of the electro-mechanical properties of single molecules. From the initial steps in point-contact spectroscopy the experiments have been refined, such that we are now able to investigate processes of elastic and inelastic transport at the scale of single atoms, and engineered atomic and molecular wires. We expect that this will continue to provide new challenges and interesting physics for many years to come.

This work is part of the research programme of the Foundation for Fundamental Research on Matter (FOM), which is part of the Netherlands Organization for Scientific Research (NWO). This work is dedicated to Igor Yanson who has been a lasting source of inspiration for our work. We thank Sander Otte for his contribution in the preliminary stages of the research.

1. Y.G. Naidyuk and I.K. Yanson, Point-Contact Spectroscopy, Vol. 145, Springer Series in Solid-State Science, Springer, New York (2005).

2. G. Deutscher, Rev. Mod. Phys. 77, 109 (2005).

3. D. Daghero and R.S. Gonnelli, Superconductor Science and Technology 23, 043001 (2010).

4. R.J. Soulen, J.M. Byers, M.S. Osofsky, B. Nadgorny, T. Ambrose, S.F. Cheng, P.R. Broussard, C.T. Tanaka, J. Nowak, J.S. Moodera, A. Barry, and J.M.D. Coey, Science 282, 85 (1998).

5. R. Landauer, IBM J. Res. Dev. 1, 223 (1957).

6. M. Büttiker, Phys. Rev. Lett. 57, 1761 (1986).

7. I.K. Yanson, Zh. Eksp. Teor. Fiz. 66, 1035 (1974) [Sov. Phys. JETP 39, 506 (1974)].

8. A.G.M. Jansen, F.M. Mueller, and P. Wyder, Superconducticity in d- and f-band Metals, Plenum, New York (1976).

9. A.G.M. Jansen, F.M. Mueller, and P. Wyder, Science 199, 1037 (1978).

10. B.J. van Wees, H. van Houten, C.W.J. Beenakker, J.G. Williamson, L.P. Kouwenhoven, D. van der Marel, and C.T. Foxon, Phys. Rev. Lett. 60, 848 (1988).

11. B.J. van Wees, L.P. Kouwenhoven, E.M.M. Willems, C.J.P.M. Harmans, J.E. Mooij, H. van Houten, C.W.J. Beenakker, J.G. Williamson, and C.T. Foxon, Phys. Rev. B 43, 12431 (1991).

12. D.A. Wharam, T.J. Thornton, R. Newbury, M. Pepper, H. Ahmed, J.E.F. Frost, D.G. Hasko, D.C. Peacock, D.A. Ritchie, and G.A.C. Jones, J. Phys. C: Solid State Phys. 21, L209 (1988).

13. G. Binnig, H. Rohrer, Ch. Gerber, and E. Weibel, Appl. Phys. Lett. 40, 178 (1982).

14. G. Binnig and H. Rohrer, Ultramicroscopy 11, 157 (1983).
15. G. Binnig and H. Rohrer, Surf. Sci. 126, 236 (1983).

16. C.J. Muller, J.M. van Ruitenbeek, and L.J. de Jongh, Physica C: Superconductivity 191, 485 (1992).

17. J. Moreland and J.W. Ekin, J. Appl. Phys. 58, 3888 (1985).

18. C.J. Muller, J.M. van Ruitenbeek, and L.J. de Jongh, Phys. Rev. Lett. 69, 140 (1992).

19. J.M. Krans, J.M. van Ruitenbeek, V.V. Fisun, I.K. Yanson, and L.J. de Jongh, Nature 375, 767 (1995).

20. Y.J. Song, A.F. Otte, V. Shvarts, Z. Zhao, Y. Kuk, S.R. Blankenship, A. Band, F.M. Hess, and J.A. Stroscio, Rev. Scientic Instruments 81, 121101 (2010).

21. attocube systems AG. URL www.attocube.com.

22. E. Scheer, N. Agraït, J.C. Cuevas, A. Levy Yeyati, B. Ludoph, A. Martín-Rodero, G. Rubio Bollinger, J.M. van Ruitenbeek, and C. Urbina, Nature 394, 154 (1998).

23. C. Sirvent, J.G. Rodrigo, S. Vieira, L. Jurczyszyn, N. Mingo, and F. Flores, Phys. Rev. B 53, 16086 (1996).

24. H. Ohnishi, Y. Kondo, and K. Takayanagi, Nature 395, 780 (1998).

25. A.I. Yanson, G. Rubio Bollinger, H.E. van den Brom, N. Agraït, and J.M. van Ruitenbeek, Nature 395, 783 (1998).

26. N. Agraït, J.G. Rodrigo, and S. Vieira, Phys. Rev. B 47, 12345 (1993).

27. J.M. Krans, C.J. Muller, I.K. Yanson, Th.C.M. Govaert, R. Hesper, and J.M. van Ruitenbeek, Phys. Rev. B 48, 14721 (1993).

28. R.H.M. Smit, C. Untiedt, A.I. Yanson, and J.M. van Ruitenbeek, Phys. Rev. Lett. 87, 266102 (2001).

29. G. Rubio, N. Agraït, and S. Vieira, Phys. Rev. Lett. 76, 2302 (1996).

30. G. Rubio-Bollinger, S.R. Bahn, N. Agraït, K.W. Jacobsen, and S. Vieira, Phys. Rev. Lett. 87, 026101 (2001).

31. J.K. Gimzewski and R. Möller, Phys. Rev. B 36, 1284 (1987).

32. U. Dürig, O. Züger, and D.W. Pohl, Phys. Rev. Lett. 65, 349 (1990).

33. J.K. Gimzewski, R. Möller, D.W. Pohl, and R.R. Schlittler, Surf. Sci. 189-190, 15 (1987).

34. L. Olesen, E. Laegsgaard, I. Stensgaard, F. Besenbacher, J. Schiøtz, P. Stoltze, K.W. Jacobsen, and J.K. Nørskov, Phys. Rev. Lett. 72, 2251 (1994).

35. J.I. Pascual, J. Méndez, J. Gómez-Herrero, A.M. Baró, N. García, and V.T. Binh, Phys. Rev. Lett. 71, 1852 (1993).

36. J.R. Smith, G. Bozzolo, A. Banerjea, and J. Ferrante, Phys. Rev. Lett. 63, 1269 (1989).

37. S. Ciraci, A. Barato, and Inder P. Batra, Phys. Rev. B 42, 7618 (1990).

38. L. Olesen, M. Brandbyge, M.R. Sørensen, K.W. Jacobsen, E. Lægsgaard, I. Stensgaard, and F. Besenbacher, Phys. Rev. Lett. 76, 1485 (1996).

39. W.A. Hofer, A.J. Fisher, R.A. Wolkow, and P. Grütter, Phys. Rev. Lett. 87, 236104 (2001).

40. S. Ciraci, E. Tekman, A. Barato, and Inder P. Batra, Phys. Rev. B 46, 10411 (1992). 
41. M.L. Trouwborst, E.H. Huisman, F.L. Bakker, S.J. van der Molen, and B.J. van Wees, Phys. Rev. Lett. 100, 175502 (2008).

42. U. Landman, W.D. Luedtke, N.A. Burnham, and R.J. Colton, Science 248, 454 (1990).

43. A.F. Otte, Magnetism of a Single Atom, PhD thesis, Leiden University (2008).

44. Y. Uehara, T. Fujita, M. Iwami, and S. Ushioda, Rev. Sci. Instr. 72, 2097 (2001).

45. U. Yxklinten, Y. Andersson, and B.I. Lundqvist, Phys. Rev. Lett. 72, 2302 (1994).

46. J.A. Ketoja, I.I. Satija, and J.C. Chaves, Phys. Rev. B 52, 3026 (1995).

47. M.R. Sørensen, M. Brandbyge, and K.W. Jacobsen, Phys. Rev. B 57, 3283 (1998).

48. W.H.A. Thijssen, D. Marjenburgh, R.H. Bremmer, and J.M. van Ruitenbeek, Phys. Rev. Lett. 96, 026806 (2006).

49. J.A. Stroscio and D.M. Eigler, Science 254, 1319 (1991).

50. L. Limot, J. Kröger, R. Berndt, A. Garcia-Lekue, and W.A. Hofer, Phys. Rev. Lett. 94, 126102 (2005).

51. D.P.E. Smith, Science 269, 371 (1995).

52. H.J. Mamin, P.H. Guethner, and D. Rugar, Phys. Rev. Lett. 65, 2418 (1990).

53. D.M. Eigler, C.P. Lutz, and W.E. Rudge, Nature 352, 600 (1991).

54. J.A. Stroscio and R.J. Celotta, Science 306, 242 (2004).

55. M. Ternes, C.P. Lutz, C.F. Hirjibehedin, F.J. Giessibl, and A.J. Heinrich, Science 319, 1066 (2008).

56. D.M. Eigler and E.K. Schweizer, Nature 344, 524 (1990).

57. S.W. Hla, K.F. Braun, and K.H. Rieder, Phys. Rev. B 67, 201402 (2003).

58. M.F. Crommie, C.P. Lutz, and D.M. Eigler, Science 262, 218 (1993).

59. L. Bartels, G. Meyer, and K.H. Rieder, Phys. Rev. Lett. 79, 697 (1997).

60. A. Kühnle, G. Meyer, S.W. Hla, and K.H. Rieder, Surf. Sci. 499, 15 (2002).
61. Y.H. Zhang, P. Wahl, and K. Kern, Nano Lett. 11, 3838 (2011).

62. M. Enachescu, D. Schleef, D.F. Ogletree, and M. Salmeron, Phys. Rev. B 60, 16913 (1999).

63. X. Bouju, C. Joachim, and C. Girard, Phys. Rev. B 59, R7845 (1999).

64. N. Nilius, M.V. Ganduglia-Pirovano, V. Brázdová, M. Kulawik, J. Sauer, and H.J. Freund, Phys. Rev. B 81, 045422 (2010).

65. T.M. Wallis, N. Nilius, and W. Ho, Phys. Rev. Lett. 89, 236802 (2002).

66. N. Nilius, T.M. Wallis, and W. Ho, Science 297, 1853 (2002).

67. L. Laerentz, F. Ample, H. Yu, S. Hecht, C. Joachim, and L. Grill, Science 323, 1193 (2009).

68. F. Pump, R. Temirov, O. Neucheva, S. Soubatch, S. Tautz, M. Rohlfing, and G. Cuniberti, Appl. Phys. A: Materials Science \& Processing 93, 335 (2008).

69. S.W. Hla, K.F. Braun, B. Wassermannd, and K.H. Rieder, Phys. Rev. Lett. 93, 208302 (2004).

70. A.J. Heinrich, C.P. Lutz, J.A. Gupta, and D.M. Eigler, Science 298, 1381 (2002).

71. J.M. Mativetsky and P. Samorí, Small 5, 1927 (2009).

72. R. Temirov, A. Lassise, F.B. Anders, and F.S. Tautz, Nanotechnology 19, 065401 (2008).

73. N. Fournier, C. Wagner, C. Weiss, R. Temirov, and F.S. Tautz, Phys. Rev. B 84, 035435 (2011).

74. M. Kulawik, N. Nilius, and H.J. Freund, Phys. Rev. Lett. 96, 036103 (2006).

75. V. Simic-Milosevic, M. Heyde, X. Lin, T. König, H.P. Rust, M. Sterrer, T. Risse, N. Nilius, H.J. Freund, L. Giordano, and G. Pacchioni, Phys. Rev. B 78, 235429 (2008).

76. S.R. Bahn and K.W. Jacobsen, Phys. Rev. Lett. 87, 266101 (2001).

77. H. Ibach, Physics of Surfaces and Interfaces, Springer (2006). 